\section{Briginal anticles.}

\section{FRACIURES OF THE OS CALCIS.}

BY FIEDERIC J COTTON, M.D.,

l'irst Assistant Visiting Surgcon, Boston City Hospital: Assistant P'rofessor of Clinical Surjery. T'ufts Collcye Medical School AND

LOU1S T. WILAON, M.D.,

Third Assistant Visiting Surgcon, Boston City Hospilal.

\section{PIREILIMINARY PAPEIR.}

Our attention was first called to fractures of the os calcis by the frequency of permanent disability, or of disability that threatened to be permanent, in cases which were seen in the surgical out-patient department of the City Hospital. It was only after seeing a considerable number of these cases that it occurred to us to observe them especially, or to treat them in any other than the routine way, which is to accept the result as inevitable. All the teachings of school and hospital, and all the data of the literature, warn us that we should be very careful to treat fractures of the os calcis by fixation and by putting them up in plantar flexion, so as to decrease the tension through the tendo achillis, which may contribute to further displacement of the fractured heel. Considering that further displacement of.the fractured heel is impossible in the average fracture of this sort, at least unless the impaction has previously been reduced, this advice is about as practical as much that stands in the textbogks.

Such treatment of fractures of the os calcis is perfectly respectable doctrine for a date ten or fifteen years ago, but is not, as we shall attempt to show, what should be expected to-day.

There are, in the recent literature, several articles that seem to show an understanding of the lesions and the relative frequency and severity of these cases. All the articles are admirable in their way. ${ }^{1}$

Exhaustive as these articles are, none of them have anything to offer in regard to what seem to us the really important points, - the recognition and proper treatment of fresh cases. T'reatment of deformity already produced by neglect does great good, but does not justify the neglect.

Up to the time when good $x$-rays could be obtained, there was no definite way of estimating the cxtent of the lesion in these cases, and in many cases it was apparently impossible even to make a diagnosis with accuracy. The general assumption in these cases was that there was a breaking across of the os calcis. It was known that complicated crushing of the os calcis might, in fact, occur, and this was shown in museum specimens, but that such was the condition in the average case seems never to have been assumed. In this, as in other fracture findings, muscum specimens were discounted on the assumption that all the lesions in a case of fatal injury were more severe than in cases ordinarily met with that recovered.

\footnotetext{
' Carless and Mayou: London Practitioner, December, 1902.

Blau: Deut. Zeit, fur Chir., 1004, lxxii, p. 445.

Schultz: Wien. Klin. Wochenschr.. 1906, xix, p. 00

J. Hutchinson, Jr.: Transactions, C̈linical Society, London, 1005, Xxxviii, p. 159.

Cabot and Binney: Ann, of Surg., January, 1907.
}

This is an obvious fallacy and, even without the evidence of the $x$-ray, it is fair to assume that the usual cause of fractures of the tarsus (namely, falls of twenty feet or over, in which the weight is received on the heels) is sufficient to furnish adequate violence for any lesion, andwe should expect a priori very severe lesions in the ordinary cases. That is, in fact, the case, and it is our belief that the lesions in all these cases are nearly alike, varying mainly in the extent and direction of the displacement of the confused fragments.

The lesion is a crushing lesion; it results, as we have said, from falls on the feet. Fractures of the bone from other causes, particularly from muscular tension through the tendo achillis from action of the calf muscles, have frequently been reported but are really only the picturesque exception. We have never seen such a case and know of but one occurring in this vicinity. This is the case at the Massachusetts General Hospital, from the skiagraph of which one of us made a tracing for the Scudder book on fractures. " There are two cases of which we have knowledge in which the fracture of the os calcis was the result of lateral crushing from a lateral blow. All other cases in our series of nearly a hundred, as in the similar series of Cabot and Binney just published, seem to have been the direct result of vertical crushing force. For our present purposes, then, we will consider only the type of fracture of the os calcis by crushing.

Before considering the lesions in detail, we will speak of results, for our inquiry has been, as it logically should be, in the line of ascertaining the proximate reason of bad results. Deductive reasoning from lesions amounts to nothing. What we really want to know in regard to the result of this or any other fracture that can be called approximately a type, is:

$A$. The results as to function.

$B$. Where there is loss of function, the proximate causes of such loss, whether from deformity, limitation of motion, weakness or what not.

$C$. How do the cases that result badly differ from the more fortunate ones, in lesion or in treatment?

D. What can be done to avoid bad results?

$E$. What, if anything, can be done to remedy bad results when they have occurred?

\section{A.}

We have done a great deal of work in the last five years trying to answer these questions and we believe that we can answer them, but regret our inability to give a statistical answer of any value to question $A$, owing to the difficulty in tracing cases. These cases are usually men in the prime of life, who, as a result of their occupation as construction artisans, roofers, painters, longshoremen, etc., have been injured at their work. Men of this sort, if they recover from injuries, usually return to work and can be traced through their unions, etc. In this series such tracing was almost always without result.

2 This is, upparently, the same case and the sume plate appearing in the recent article of Cabot and Binney. 
We are convinced that a very large percentage of cases of this sort result in permanent disability sufficient at least to prevent resumption of the man's ordinary work. We believe that the endresults we have been able to study probably represent somewhere near the average of cases and that they certainly do not present a view more pessimistic than the facts. 'This we mention at some length because our impression in the matter is somewhat at variance with that expressed in the admirable paper lately published by Cabot and Binney.

We belicve the results detailed and implied in their article are much too favorable. So far as we can estimate from our own observations, reliable for end-results in 22 cases and covering a total of over 90 cases, we should estimate the proportion suffering from considerable permanent disability as certainly a good deal over one half of the total number.

\section{B.}

CAUSBS OF DISABILITY.

In studying the disabled eases we have found the following conditions of importance:

1. Ratising of the heel.

2. Shortening of the heel.

3. Outward deviation of the hecl.

4. Flattening of the arch.

5. Projection of fragments into the sole of the foot.

6. Loss of motion between the astragalus and the os calcis.

7. Mechanical interference between os calcis and external malleolus.

1. Raising of the hecl produces a shortening, ordinarily of no. great consequence. If the heel is simply driven upward, it produces no flattening of the arch; it simply raises, so to speak, one of the abutments of the arch. It does, however, bring the top of the arch close to the ground and may, in a foot originally having a low arch, cause some pressure of the soft tissues, including the nerves, between the bony arch and the sole of the boot. Fxtreme displacement may, of course, entirely destroy the arch. This condition we have seen in three cases in which relief was possible only with an artificial pad under the heel which relieved symptoms and confirmed the diagnosis as to the cause of this pressure pain.

2. Shortening of the heel per se, that is, simple approximation of the insertion of the tendo achillis to the ankle joint, has no ill effect excepting in so far as it shortens the lever arm on which the calf muscles act. T'his shortening must obviously occur, but the proof of any severe disability from this cause is lacking so far as our direct evidence goes. Some loss of power is present in all cases of this fracture, but apparently not in direct proportion to this shortening.

3. Outward deriation of the heel. - If the heel is driven outward as well as upward, the whole base of support of the foot is entirely shifted and the occurrence of flatfoot from strain alone is as nearly incvitable as in a case of Pott's fracture with uncorrected outward displacement. In one case observed by us this was the essential cause of a practically total disability. In several other cases it was a part cause.

4. Flattening of the arch.- Flattening of the arch in the sense of a simple pes valgus,. dependent on weakness alone or as a result of weakness plus the strain of accommodation to loss of motion in the ankle, may occur in these cases exactly as it occurs in Pott's fractures or in fractures higher up in the leg, entirely independent of displacement in any direction and entirely independent of the exact type of the original lesion.

The distinction between these cases and the flattened foot belonging to Classes 1 and 3 is practical as well as definite. Cases of this type, 4 , can be treated and relieved, like an ordinary pes valgus, with plates. Classes 1 and 3 are respectively made worse or are unaffected by plates.

5. Projection of fragments into the sole. (See Fig. 3.) - As a result of extreme tilting of the posterior fragment, or of a particularly sharp point on said fragment, or of an excessive callus formation there abouts, we may have a bony projection into the soft tissues of the sole of the foot. This acts exactly as does a like projection from whatever cause that pushes down into the sole, pressed down by the body weight. This was the main cause of disability in two of our cases; in one of these it was removed by operation.

In one case it seemed that a rotatory displacement of the os calcis which brought the weight on one instead of both tuberosities was the cause of the great soreness in the sole.

6. Loss of motion between the astragalus and os calcis.- This proved to be a very variable but a very serious item in causing loss of function. It is a known fact that the rotary motion by which the foot adapts itself to inequalities of the ground is a rotation of the foot as a whole underneath the astragalus. ${ }^{3}$ We also know that loss of this motion in this case mainly from adhesions, etc., is a very serious factor in the disability often following fractures at or above the ankle. ${ }^{45}$ It is, in fact, a distinctly more serious disability than limitation of the hinge motion at the ankle-joint itself. In examining these fractures of the os calcis we found this limitation of motion present not only in old cases but in other cases in which the injury was seen immediately or within less than a week, cases which, therefore, could not have suffered from long fixation. Moreover, in old cases showing such limitation of motion, it is not associated with any limitation whatever in the vertical motions at the ankle-joint. It occurs in some cases to marked degree; in others not at all. In one patient with double fracture there was before operation an absolute loss of lateral motion on one side, no such loss whatever in the other foot. Early in our observations it became clear that this limitation of lateral rotation was a very s R. W. Iovett and F. J. Cotton: Trans. Am. Orth. Asso., 189s. vol. xi, p. 298; and Boston Mro. ANo Suro. Joun., 1898, exxxix, vol, xi, p. 298; and Boston Men. AND Suko. Jouk., 1898, exxxix, ' F. J. Cotton: Boston MEn. AND Suna. Joun., 1905, eliii, R. Hon. chusetts Medical Society, 1005. 
important point in determining disability, but it was only after some study of the x-rays that we discovered the reason, namely, an involvement of the posterior joint between the astragalus and os calcis, the gliding motion in which is essential to,rotary motion of the foot.

That damage to this joint plays a part, we have had the opportunity to demonstrate by operation. lixactly what happens in all cases is hard to say. We believe, however, that the cases in which this motion is lost are those in which a fracture traverses the posterior articular surface of the calcis, or otherwise throws the joint hopelessly out of line or contour. Sometimes fracture involving the sustentaculum tali may contribute to this loss of motion (see lig. 2).

Direct involvement of the joint by fracture is obvious in Fig. $4(d)$. Indirect involvement by shifting of the relations between joint surfaces is competently shown in Fig. 3, and Fig. $4(b)$.

'The question whether or no the line of fracture directly invades the joint may or may not be shown by the skiagraph. The fact of functional interference with this joint may fairly be inferred from the loss of motion in this joint alone, unaccompanied by any loss of motion elsewhere in the foot or ankle. Such loss is, as we have shown, an immediate result of the fracture.

7. Mechanical interference between the os calcis and the external malleolus. - One of the common complaints after the patient begins walking, or trying to walk, is that there is severe pain, located just below the external malleolus. 'There is in all of these cases a marked bony thickening on the calcis below the malleolus. This is not edema or even callus, but is due to a displaced shell of bone, consisting of the relatively thick outer layer, apparently always split off and always displaced outward. Where it projects far outward, the external malleolus comes in contact with it and the peroneal tendons play across in altered surface. The condition is not unlike the similar contact between fibula and os calcis, established in some of the extremest cases of flatfoot. The trouble is not relieved by support of the arch in the fracture cases, though relievable by the use of the chisel, as we have had an opportunity to demonstrate.

The foregoing seem to be the factors which operate as causes of disability in these fractures. We have not found that shortening (from the knee to the ground, which is always present in some degree) is of any consequence. We have not found any loss of dorsal flexion, to say nothing of any disability caused by such loss, and we have not found any such interference with the function of the calf muscles as would be responsible for any real disability beyond possible clumsiness of gait.

$C$.

As to the differences in anatomical lesions, in cases with one or another of the above-noted causes of disability, there is not a great deal to be said. We do not believe that it is possible to classify os calcis fractures according to fracture lines. Even with the $x$-ray it is very hard to trace these lines, and when we have traced them, we may be sure that there are other lines in other planes which we have not seen, and our conclusions as to details are of little value. The sketches here submitted have been selected as representative of 35 directly at hand. 'They have been drawn to show all that can be made out with certainty from the plate, including much more detail than is possible in the reproduction of a print. (See Fig. 4.)

All our attempts at classification have failed. There is a general similarity, general only. 'The central point of the fracture, so to speak, usually lies below the posterior part of the astragalus. From this point lines, of ten in threc directions, radiate through the bone. The variety of position and direction of these lines is obvious from the figures. Usually there is some diminution in the vertical depth of the bone, accompanied, as we know, by a transverse widening. Displacement of the heel upward may result in very various changes in relation of the individual fragments.

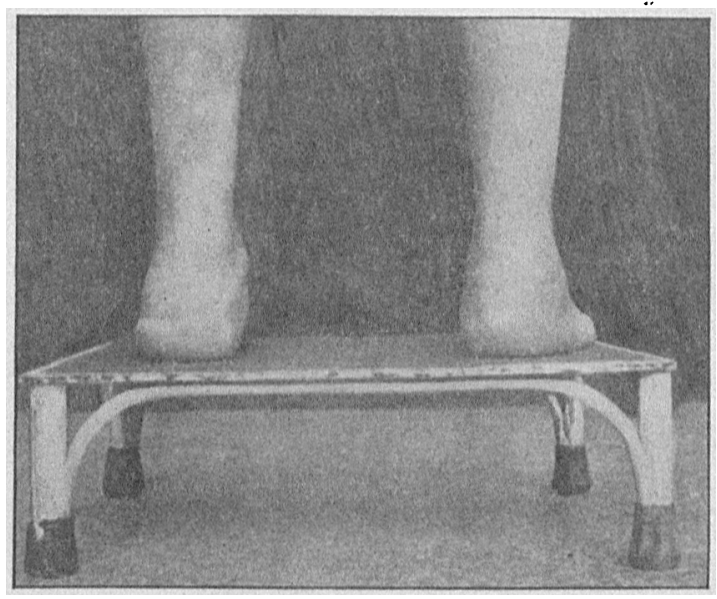

Fro. 1. Back view of an old case of double fracture of the os ralcis, right und lett. The loss of prominence of the tendo achillis is actual, not a defert of the photograph. The let
foot shows well the thickening below the external malleolus.

From the $x$-ray we may say something about the amount of upward displacement of the heel, but this is accurate only in case $\mathrm{x}$-rays are taken of both feet and compared. We may judge something of the shortening of the heel anteroposteriorly, a relatively unimportant matter. We may say with some probability what the chance is of trouble from the projection of fragments downward. Figure 3 , for example, must almost inevitably give some trouble from the projection of the sharp spur shown. It may be that we can sometimes say from the skiagraph whether or no the posterior joint between astragalus and os calcis is damaged. (See ligs. 3 and 4.) This is, however, not always easy to make out with certainty.

'To sum up, the $x$-ray tells us about spurs in the sole and (properly used) tells us about the amount of vertical displacement.

Our other data must come from physical examination. Measurements we have found of no 
use. The only measurement that is even accurate is a vertical measurement from the internal malleolus to the bottom of the heel and this tells us nothing not otherwise obtainable. Examination to estimate the vertical displacement is, owing to the swelling of the parts, too deceptive to be worth anything. In this regard we have come to trust the $x$-ray entirely, always using the skiagraph of the other foot as a " check."

Displacement of the heel outward may, on the contirary, be recognized with some accuracy by the touch. It is usually possible to make out pretty clearly the two lateral surfaces of the os calcis at the back, which should converge slightly upward toward a vertical line. If the whole heel is shoved outward or twisted in either direction, it is, therefore, usually possible to make the displacement out.

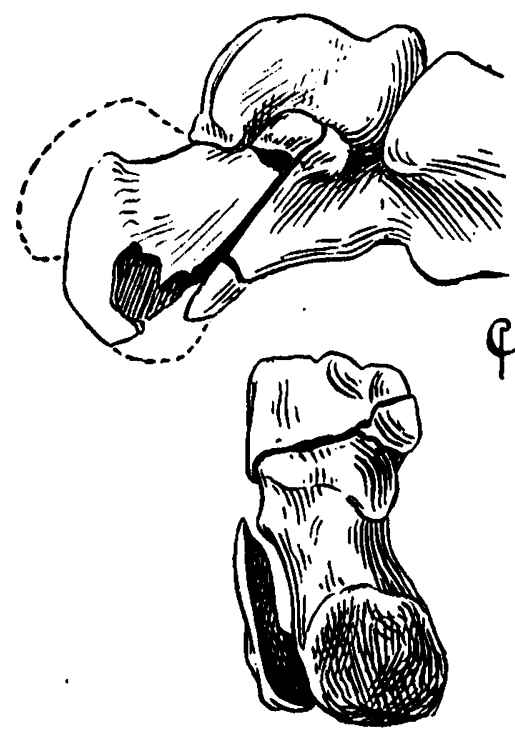

Tho. 2. Drawn from an autopsy specimen. The dotted line shows above the position of original displacement. The dotted line below slaws the bone outline where fragments have been lost.

One more point, important in prognosis, is also of diagnostic importance; namely, the thickening below the external malleolus. 'This is the best diagnostic point we have by which to recognize the prescnce of os calcis fracture. In any case, no matter how swollen, it is possible to make out this thickening and to make out that it is at least in part bony and to estimate its projection outward." In the sevorest cases this projection is so great that the normal prominence of the external malleolus has clisappeared, not only from sight, but from touch, so far as any prominence is concerned. Bony projection definitely palpable below the malleolus has been present in every case we have examined of crushing fracture of the os calcis, whether fresh or old. It has been present in several cases in which the $\mathrm{x}$-rays were doubtful, in which forced manipulation proved the fracture

- The broadening of the heel is ordinarily described as if it were generul. In fact, it is usually essentially limited to the outer side. Only where there has been involvement of the sustentaculum tali in the fracture, or where the smushing and broadening has been extreme, do we get palpable changes in bony contour to the inner side.
(See Fig. 1.) by showing marked crepitus. It was absent in one case of cross fracture only. The origin of this projection is readily seen on examination of a fresh specimen obtained by one of us (Fig. 2) in which may be seen how the outer plate of the bone, the cortical layer, is split clean off. When this specimen was dissected, the outward displacement of this plate was very marked, the interval between it and the crushed central mass of bone being filled with bony and other detritus.

We may say, then, that it is practicable by clinical examination, aided by the $x-r a y$, to determine all of the displacements which are of importance to prognosis. It is our belief that these displacements in their varying degree and direction determine the gravity of this injury. It is not our belief that there is any great difference in the fracture in different cases other than that of displacement and of minor details of fracture lines, or that we can divide these cases of crushing fracture of the os calcis into any definite types of clinical value.

\section{D.}

What can be done to avoid bad end-results?-We all know that the ordinary trentment is a purely expectant one. Under it nothing is done except to prevent increase of displacement. It is obvious that the proper treatment theoretically is to correct the displacement, but attempts at such correction are met by three distinct difficulties, and for the five years that we have been at work on this problem we have, until lately, had no answer to the question in hand. 'To-day we believe we have the answer and it is quite as much with regard to the surgical treatment of fresh cases as with regard to the remedying of old disability that we wish to call your attention to these fractures.

The difficulties met with in handling fresh cases are:

1. Difficulty in correcting displacement, owing to swelling and the difficulty of getting a firm grip on the os calcis.

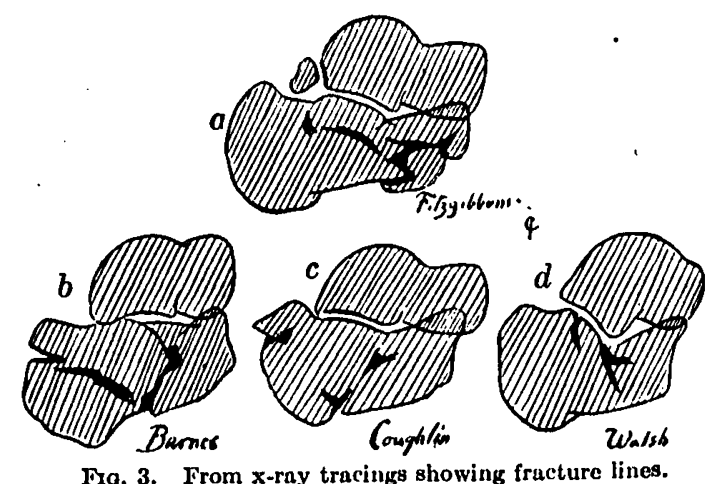

After experimenting with the Thomas wrench, with Adams' modification of the same, and with the heavy wrench used by plumbers, we have found that none of these are more efficient for this particular purpose than the unaided hand. In some cases it is possible with the thumbs and fingers to get a very satisfactory grip behind the 
tendo achillis, a grip sufficient to enable one to break up the impaction always present, and to reduce the fracture to the desired position. In other eases, lateral wrenching with the hands will break up the impaction, and then a direct pull will give the proper position.

In still other cases, this sort of grip is insufficient. In these cases it is practicable to make small incisions (after aseptic preparation and under ether) on the inside and outside of the ankle just in front of the tendo achillis and to pass a steel sound (20 to 25 French) from side to side in front of the tendon and in contact with the upper surface of the bone. 'This gives a perfectly satisfactory grip for any manipulations desired. Regard will naturally be paid to asepsis, but the danger of sepsis is small, and, even at the worst, infection of this wound would not involve the fracture, as it is in no direct relation to it. We have used this maneuver repeatedly and have never had the least trouble from it in any way.

In exceptional fresh cases with great broadening we have used the felt and mallet, striking the outer sicle of the bone, as a means of correcting displacement where general loosening up was not called for by any displacement of the heel. We do not recommend it, however, as a routine.

2. Difficulty in retaining position of fragments after reducing the impaction. Tenotomy is confidently recammended. We have tried it and found it useless and unnecessary, save, perhaps, in exceptional,cases for exceptional.reasons. All that tenotomy will do may be accomplished by a very moderate plantar flexion at the ankle. In fact, the displacement of loose fragments depends less upon the pull of the heel cord than on the almost unavoidable pressure of the heel on the bed or on the plaster bandage.

To maintain correction, we must pin or suture the fragments or reimpact them.

Any maneuver involving incision in these cases, while we still have comminuted fragments and blood clot, is too risky to be advocated.

Reimpaction sounds impracticable. In fact, it is easy. We discovered how to go about it in the course of attempts to drive back the outwardly displaced outer cortical layer. In trying to accomplish the reduction of this outward spread, we found it possible to produce, by heavy lateral pressure, a reimpaction of the loose fragments into the shape desired, sufficiently firm to maintain the new relation of the fragments provided no strain is put upon them. This impaction may sometimes be accomplished by the pressure of the hands, driving the fragments in laterally. Ordinarily we have placed a firm sandbag under the inner side of the foot and then, protecting the tissues with a folded felt pad, have used a heavy mallet to reimpact the thoroughly loosened and replaced fragments in the desired position. (See Fig. 5.) Reimpaction is not absolute, and it is possible that increase of comminution may occur under this manipulation, but this not does matter.

$\mathrm{Up}$ to date, in a considerable number of cases, we have not failed to get a satisfactory impaction, good enough to hold the fragments where they were put. Nor have we had any untoward results; the reaction to a handling which seems brutally rough, is almost nil; rarely is there even any pain or any appreciable increase of swelling.

There is one more important point, namely, the restoration of lateral motion of pro- and supination of the foot. The loss of this motion has often been noted but no suggestions ever made as to removal of the disability.

We have found, as alove noted, a great frequency of this disability but no regularity as to its mode of production. The posterior calcaneoastragaloid joint may be " smashed " or luxated or subluxated and there seems no way of determining just what has happened. Accordingly we have gone ahead in the common cases, where this loss of motion persists after reduction of the impaction and the obvious displacement, by forcing pro- and supination till a fair degree of motion was secured. Hxactly what happens it is hard to say. It is, at all events, fair to conclude that it is better to leave the joint with free motion than to leave it with none, even if some of this

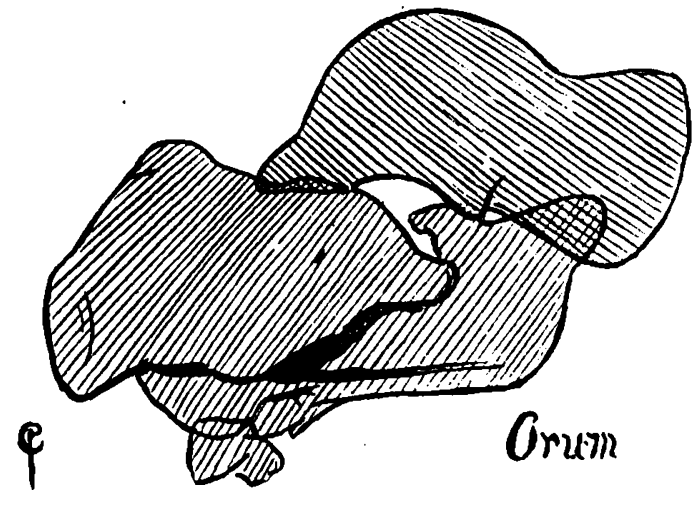

Iia. 4. From the x-ray. Shows how frngments muy form a spur.

motion may perhaps be lost later. In our cases it has not been lost later and we reckon this part of the reduction as very important in securing a good functional result. In ten fresh cases good lateral motion was secured by such manipulation where there had been none, or next to none, before this manipulation.

It seems, then, with these new methods of handling, that the old data as to the uselessness of interference must be abandoned. Given a case of fracture of the os calcis with any displacement, with loss of motion, with marked bony thickening below the outer malleolus, or with spurs, or the prospect of spur formation, toward the sole, it is our duty not to sit by idly, but to interfere. And our interference should occur before any considerable repair has gone on, usually at three to seven days by choice. The routine should be: Ether. Breaking up impaction (with hands alone or with a spindle inserted beneath the heel cord). Correction of any upward or lateral displacement. Securing of adequate motion between calcis and astragalus by forced pro- and supination. Reimpaction with sand-bag and mallet or by manual pressure. 
AFTLRT-TIRLA'TMEN'T.

The apparatus used in after-treatment in our cases has been an ordinary " cast" of plaster of Paris equipped with two felt pads, one over the tendo achillis and os calcis, one over the dorsum of the foot. After the plaster is on, the foot is put up at about 15 to $20^{\circ}$ of plantar flexion, and during the setting of the plaster, downward traction is made with the hands on the dorsum of the foot and behind at either side of the tendo achillis. Usually it seems wise to secure some inversion as well as very slight plantar flexion. The reaction following such reduction is surprisingly slight. 'The patient cin be up on erutches (if the lesion is of one side only) within a week. In a week or ten days the primary callus seems adequate to hold the comnection. At this time removal of the cast occasionally for massage and guarded active motion is wise. We have not allowed weightbearing for a month, though delaying the beginning of use so long may be unnecessarily conservative. 'There seems little tendency to

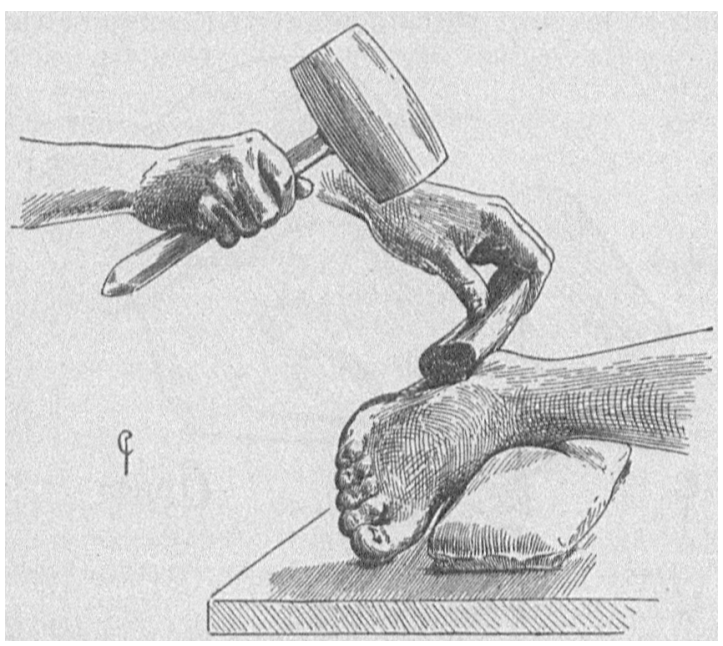

Fis. 5. Reimpaction after reduction.

reproduction of deformity or stiffening. Return of full function is rather slow; probably three months will be a fair average of time.

The cases treated in this way have all done notably well; they are too few to form a sound basis for argument, perhaps, but there is no question in our minds that the results are incomparably better under this routine and that rational procedure along these lines should take the place of the utter negligence that has passed for treatment in these cases.

\section{$E$.}

Remedies for bad end-results. - The detailed conditions relating to disability in this fracture have been considered. Corresponding to these, and not in any routine fashion, we may apply the remedy which is mainly, though not always, an operative remedy. If the disabling condition is one simply of pes valgus, we may apply metal plates or felt pads with some success, or may use the 'Thomas sole, of ten of excellent service in these cases and sometimes better borne than plates because it avoids any direct pressure under the arch. Several years ago, when we first began to look into these cases, Dr. E. G. Brackett very kindly assisted us in trying palliative measures. It is because we have seen his thoroughly expert work on this early series that we feel confident in saying that it is only the cases of uncomplicated static pes valgus following this fracture that can substantially be benefited with support, while direct flattening of the arch and pes valgus from outward displacement of the heel are not remediable by apparatus. To some slight extent, plates or a Thomas sole will relieve the friction of the external malleolus on the os calcis and to some extent pads will relieve the pressure on the soft parts under the flattened arch, but these pads, to be effective, should not be placed in the arch but under the heel ilself. Attempts to remedy pain from pressure of bony spurs in the sole by shifting the weight to other points with a plate have been of only very slight use as a rule.

Of the causes of disability not remediable by apparatus, we have:

Loss of motion in the joints.

Fxcessive bony thickening below the external malleolus.

l3ony spurs in the sole.

Outward displacement of the heel.

Extreme upward displacement of the heel.

'These conditions are all essentially operative. The operations we have done for them have been as follows:

In five inveterate cases forcible correction was donc, under ether, with early passive motion, to restore lost lateral motion in the subastragaloid joints. One of these cases was a year old. In all cases the result was excellent; in fact, it is surprising that this scheme should work so well as it does in old cases.

Excessive thickening below the external malleolus we have treated in only two old cases. The treatment in these cases was chiseling off a considerable portion of the outer side of the bone through an incision behind and below the ankle. There seems to be no argument against this operation and no risk involved. There is no technical difficulty except that some of the thickening lies under the peroneal tendons, the sheath of which must be preserved. In one of the cases operated there was almost entire disappearance of the previous disabling pain and there was no evidence of any tendency to recurrence of bone growth. The man was able to resume work after over a year of idleness and has continued up to date. The other case showed some improvement after a month, but was lost track of, so that the permanent improvement cannot be stated.

Bony spurs in the sole we have removed in three cases only. In one case painful pressure at the site of a spur shown in an $x$-ray produced total disability. The spur was removed with a chisel through an incision just above the outer edge of the sole with the result of greatly improved approximately normal function. The second 
case was similar in details and results; the third is too recent to admit of report as to results.

Outward displacement of the heel we have dealt with but once. This was a case operated on by one of us in 1903 for extreme upward, as well as outward, displacement. Operation was done two months after injury to relieve practically total disability due to extreme flat foot combined with pain from pressure underneath the flattened arch. In this case the operation done was Gleich's operation for flat foot. A cross section of the os calcis behind the joint was executed with a chisel through lateral incisions. Probably the Gigli saw, then not available, would work better. 'lihe heel was then displaced downward about three quarters of an inch, inward about the same distance. 'Tenotomy of the tendo achillis was done and the position retained by plaster. At the end of five weeks he was already walking better than before the operation and was discharged with the os calcis firmly united in its new position. We have been unable to trace him since and can give no further data.

\section{CONCLUSIONS.}

The points we would wish to emphasize in this preliminary paper are as follows:

Comminuted fracture of the os calcis is relatively common.

Nearly all cases are of substantially the same type.

'The results' are rather frequently' so imperfect as to interfere seriously with work or to disable the sufferer entirely.

The cause of disability is not the fracture as such, but certain displacements, outlined alsove, which should be remediable or rather preventable.

The treatment ordinarily advocated and carried out is no treatment at all. We have all of us been guilty of what is very nearly criminal negligence in regard to these cases.

Certain measures are practicable in the treatment of fresh cases that will minimize, or of ten prevent, the special displacements which are the cause of disalility. It is our cluty to adopt a routine embodying some such measures along the lines of the routine above suggested.

Provided such measures have not been carried out, and provided disability is present, palliation is possible by apparatus in some cases, while in others operations of no great gravity or technical difficulty will insure decided improvement of function.

This paper is, then, a plea for more accurate study and more active treatment, a plea, in other words, for a real surgical treatment for this class of lesions.

THE first lecture in the Harvey Society course of 1908-1909 was given on Oct. 24, at the New York Academy of Medicine, by Prof. A. Calmette, of the Institut Pasteur de Lille, France, on the subject, "Intestinal Infection and Immunity in Tuber-
THE ETHMOID CELLS AT BIRTH AND THEIR DEVELOPMENT DURING FETAL LIFE.

BY E. J. CUHAN, M.1., BATHURST, AUBTHALIA.

(From the Larynoological Department of the Harvard Medical School.)

THe object of this paper is a study of the ethmoid labyrinth at birth and its development during fetal life, with regard to the number, size, position and state of development of its cells. In order to gain an adequate idea of the condition to be found, and to realize the importance of a consideration of the development of the air sinuses at this stage in connection with what they have been and what they will afterwards become, it is necessary to review briefly some points in the anatomy of these structures in the adult, and to follow certain stages of development in the fetus.

The ethmoid bone lies in front of the sphenoid and between the orbital plates of the frontal. Its outer walls, the ossa plana, help to form the inner walls of the orbit. 'The ethmoid may be said to consist roughly of three parts: 'Two lateral masses made up of air cells with thin walls and turbinates, and the vertical plate, lamina perpendicularis. These three are united above by the cribriform plate which lies in a plane at right angles to them. 'The vertical plate hangs down between the lateral masses and forms the upper part of the nasal septum. There is a prolongation upward into the cranium, the crista galli, to form an attachment for the falx cerebri. The lateral mass, when in situ, hangs down on either side of the septum and forms part of the outer wall of the nasal cavity. Each is an approximately wedgeshaped mass, honeycombed with many air cells, the walls of which are made up of very thin lone and lined with thin ciliated epithelium with a small number of mucous and serous glands. 'The long axis of this prismatic-shaped mass is anteroposterior, and the apex downward, the base being attached to the cribiform plate. Externally, the smooth orbital surface of the ethmoid, the os planum, roofs in some air cells. It articulates above with the orbital plate of the frontal, which also roofs in external air cells. In front, the os planum articulates with the lachrymal bone and below with the orbital surface of the superior maxilla. Posteriorly, it articulates with the sphenoid, and postero-inferiorly with the orbital process of the palate bone, both of which serve to roof in some of the ethmoidal cells.

On the mesial surface, we have two, and sometimes three, turbinates (the inferior turbinate loes not belong to the ethmoid bone). If there are two turbinates, there are also two meati, the middle meatus and the superior meatus, but if there happens to be a third turbinate, the space under it is called the meatus supremus. In the superior meatus, there are openings for the posterior ethmoidal cells and the sphenoidal sinus. If the meatus supremus is present, the sphenoidal sinus enters it, but if not, it drains into the vestigial fossa of this meatus.

The ethmoidal cells are divided into two groups, namely, (1) the posterior, which drain into the superior meatus, or into the space above the 